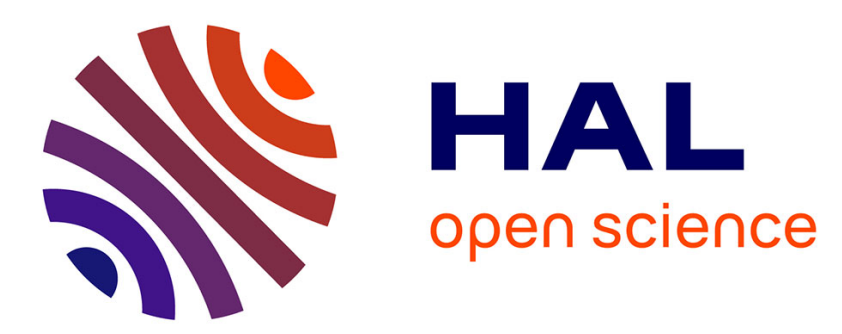

\title{
Augmented Reality Application in Manufacturing Industry: Maintenance and Non-destructive Testing (NDT) Use Cases
}

Fakhreddine Ababsa

\section{- To cite this version:}

Fakhreddine Ababsa. Augmented Reality Application in Manufacturing Industry: Maintenance and Non-destructive Testing (NDT) Use Cases. 7th International Conference on Augmented Reality, Virtual Reality, and Computer Graphics, Sep 2020, Lecce, Italy. pp.333-344, 10.1007/978-3-03058468-9_24. hal-02976923

\section{HAL Id: hal-02976923 \\ https://hal.science/hal-02976923}

Submitted on 23 Oct 2020

HAL is a multi-disciplinary open access archive for the deposit and dissemination of scientific research documents, whether they are published or not. The documents may come from teaching and research institutions in France or abroad, or from public or private research centers.
L'archive ouverte pluridisciplinaire HAL, est destinée au dépôt et à la diffusion de documents scientifiques de niveau recherche, publiés ou non, émanant des établissements d'enseignement et de recherche français ou étrangers, des laboratoires publics ou privés. 


\title{
Augmented Reality Application in Manufacturing Industry: Maintenance and Non-destructive Testing (NDT) Use Cases
}

\author{
Fakhreddine Ababsa ${ }^{(凶)}$ \\ Arts et Metiers Institute of Technology, LISPEN, HESAM University, Paris, France \\ Fakhreddine. Ababsa@ensam. eu
}

\begin{abstract}
In recent years, a structural transformation of the manufacturing industry has been occurring as a result of the digital revolution. Thus, digital tools are now systematically used throughout the entire value chain, from design to production to marketing, especially virtual and augmented reality. Therefore, the purpose of this paper is to review, through concrete use cases, the progress of these novel technologies and their use in the manufacturing industry.
\end{abstract}

Keywords: Augmented Reality · Industry 4.0 • Manufacturing · Human-machine interaction $\cdot$ Maintenance and quality monitoring

\section{Introduction}

We are in the era of the fourth industrial revolution after that of mechanization, massproduction and automation. Thanks to the digital transformation, factories are more and more connected and appear as part of a global ecosystem that includes the entire production chain. This new paradigm is called Industry 4.0, it aims to maintain and develop a strong and innovative industrial activity. Factories become smarter, more competitive because they are more efficient and safe; their production processes are completely changing. This transformation is mainly based on new technologies such as robotics, virtual and augmented reality, sensor networks, Internet of Things (IoT), data processing, big data, etc. It is a new model that puts the human at the heart of business and organization. Thus, the operator in his factory will accomplish his daily tasks in a different way; he will be equipped with wearable and intelligent devices that allow him to communicate and collaborate with other operators, and to assist him in carrying out his task. At the same time, customer expectations are changing much faster than before. To cope with this, companies need to be able to detect weak signals and feedback this information to anticipate changes in customer behavior. They must also be able to innovate much more quickly, to adapt their offer by adopting an agile organization allowing flexible and therefore completely reconfigurable production modes [1], able to deliver customized and competitive products. Virtual and Augmented Reality is one of the key components in achieving the goals of the industry 4.0. For example, virtual reality is used during the product creation process to optimize and prototype experiments in order to test new 
products "in near-use situations". It also allows employees to receive hands on training thanks to virtual environments, which mimic production equipment in a very realistic manner [2]. This prevents expensive down time and disruptions in normal operations, and improve the efficiency of skills transfer and increased knowledge retention. On the other hands, augmented reality is a powerful tool that allows operators to better undertake tasks on site, thanks to its ability to visualize virtual objects in the real word. This is the case for tasks such as parts assembly [3, 4], maintenance [5], quality control or material management [6]. The goal of this paper is to show the practical uses of augmented reality in the manufacturing industry. We will present two innovative solutions to answer the maintenance problem encountered in the manufacturing industry. The paper is organized as follows. Section 2 describes the related projects. Section 3 introduces the Key technologies on augmented reality currently used in the manufacturing industry. Section 4 shows two practical applications of this technology and provides a detailed analysis of its usability in an industrial setting. In Sect. 5, we present the conclusions and future work.

\section{Related Works}

The use of Augmented Reality in industry goes back to the 1990s. In 1993 [7] Boeing engineers presented one of the first see-through displays integrating a head position estimation approach and a real/virtual registration system, and allowing to increase the operator's vision with digital information relevant to the job he is performing. On the other hand, several companies (EADS, BMW, Airbus, etc.) participated in the ARVIKA project (1999-2003), which aimed to study the capacity of augmented reality to support work processes in the design, production and service for complex industrial products and plants $[8,9]$. The project STARMATE, funded by the EU (2000-2003), provided two main functionalities: User assistance during assembling task of complex mechanical parts and increasing the operator's skills to carry out such processes by passing them training scenarios in augmented reality. The ARTESAS project led by Siemens (2004-2006) aimed to develop an augmented reality system for aeronautical and automotive maintenance [10]. AVILUSplus (2009-2011) is a collaborative project that brought together German basic industries and research institutes to develop innovative systems in the areas of Virtual and Augmented Reality for products life cycle (PLM) services. The main areas are information management, simulation and rendering, tracking, interaction and geometry acquisition [11]. Testia, a subsidiary of Airbus, has developed the SART (Smart Augmented Reality Tool) system for the aerospace industry. A system that allows inspection personnel, as part of quality control, to establish a digital model of the actual part on a tablet or PC, and thus reliably identify non-conforming parts [12]. The European project REPLICATE [13] has improved the human creative process by integrating new user experiences in mixed reality. Thus, experimental solutions such as 3D/4D story-boarding in unconstrained spaces and the expression of new ideas by manipulating objects (assembly/disassembly) in a co-creative workspace, have been developed and tested in an industrial context. In the same way, the European project SPARK (Spatial Augmented Reality as a Key for co-creativity) (2016-2019) aims to realize an intuitive ICT platform based on spatial augmented reality (SAR) in order to show designers and customers new products/concepts in the form of mixed prototypes during brainstorming sessions, with the 
aim of enriching the design process. It is a collaborative platform that allows users to work freely together, promoting open innovation and creative thinking [14]. In 2017 Safran Landing Systems launched the Argo (Augmented Reality for Ground Operations) project [15], which aims to deploy augmented reality on an industrial scale at Safran's various MRO sites to help operators with aircraft maintenance tasks and automate the writing of technical operating reports. In this way, Safran hopes to reduce intervention times, which would give a considerable competitive edge as an increase in air traffic goes hand in hand with an increase in MRO activities. In addition, several research works have been conducted over the last years to develop AR systems dedicated to the industry. For example, in [16] the authors propose the ACAAR system (Authoring for Context-Aware AR) which allows users to add and organize digital content (text, images, CAD) in 3D space and specify its relationship with the maintenance context. In [17] the authors give a thorough state of the art on the use of AR in industrial maintenance. Future research directions are also proposed, including hardware, monitoring and user interaction. Other studies, such as in [18], have focused on the use of mobile devices such as smartphones and tablets to perform maintenance and assembly procedures with augmented reality (AR). They evaluated performance by comparing the execution times and errors made when performing a maintenance procedure with an augmented reality tool and paper-based instructions.

\section{Augmented Reality Technologies for Industrial}

\subsection{Applications}

Traditionally, augmented reality has been seldom used in the manufacturing industry due to the lack of maturity of the technology, computational and hardware were limited and visualization devices uncomfortable. The advent of tablets and smartphones (Fig. 1) with more capabilities have enabled the development of the first augmented reality applications in industrial environments.

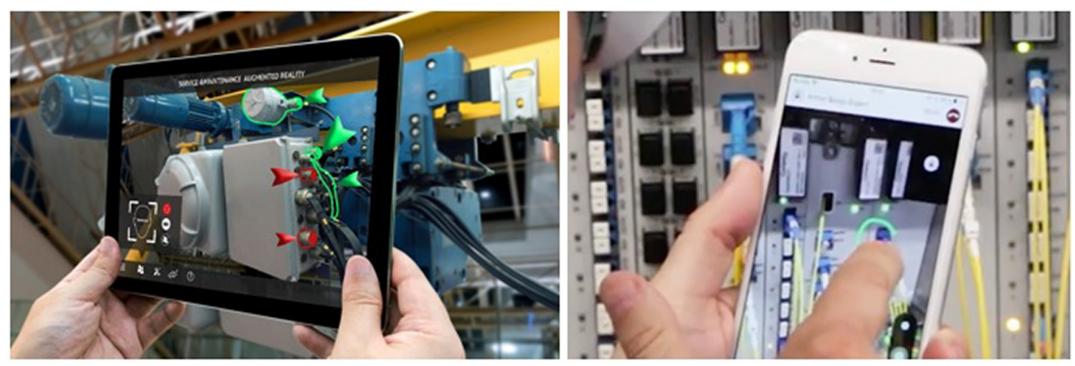

Fig. 1. AR mobile device

However, these devices have not been as successful as expected, as they can divert attention of the operators, who need to have their hands free to perform their tasks. The head-mounted displays (HMDs) developed in recent years overcome these limitations. 
The operator equipped with such a device can view spatialized information/instructions in hands-free mode while performing his task. In addition, these helmets over automatic gesture recognition allowing interaction with 3D holograms. Furthermore, the performance of HMDs continues to improve with each release, offering increasingly attractive functionalities for creating immersive and personalized user experiences, which makes it easier to integrate them into industrial processes [19]. This explains the new craze for Augmented Reality in the industry in recent years. HMDs are now able to detect information coming from the environment and process/interpret them in real-time. They are more comfortable, less heavy, and easy to use and have a larger field of view.
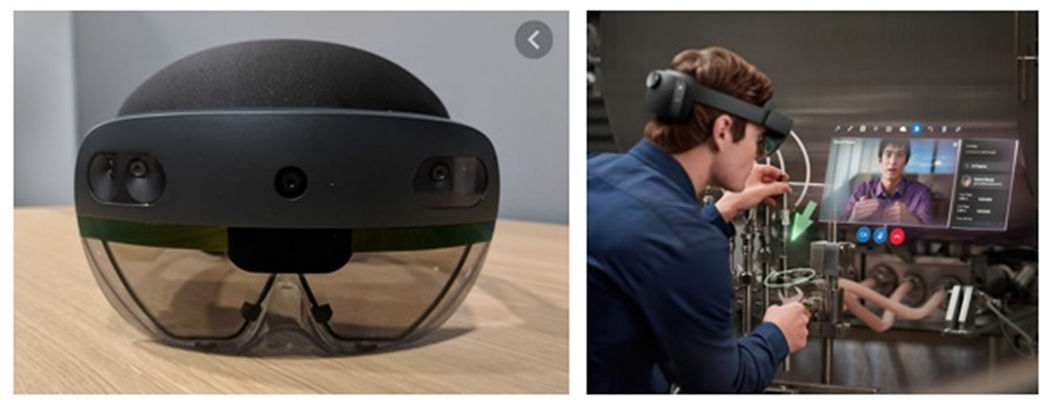

Fig. 2. Microsoft HoloLens 2

Figure 2 shows the Microsoft HoloLens 2 headset released in 2019, which has the following characteristics:

- A diagonal field of view (FoV) of $52^{\circ}$, improving over the $34^{\circ}$ field of view of the first generation of HoloLens headset, while maintaining image fidelity at 47 pixels per degree of sight. In practice this, will ensure a better user experience.

- An integrated finger and hand movement recognition and tracking system with greater accuracy. This is what makes it possible for users interact directly with $3 \mathrm{D}$ content with more natural gestures.

- A powerful eye tracking software, allowing users to interact efficiently with the GUI.

- The battery is made of carbon fiber and therefore lighter compared to the HoloLens 1 , it is positioned behind the head. It allows 2 to $3 \mathrm{~h}$ of active use. The total weight of the helmet is $566 \mathrm{~g}$.

- A voice-based interaction thanks to command and control on-device and Cortana natural language with internet connectivity.

Thanks to all these improvements, the Hololens 2 headset is now considered the reference device for the implementation of augmented reality applications in industrial environments.

\subsection{Programming Library}

The development of an industrial application based on augmented reality requires the use of high-performance software capable of tracking 3D objects, reconstructing and 
understanding the often cluttered and changing work environment. The most frequently used libraries are:

- ArtToolKit: open-source marker-based video tracking library that displays virtual 3D models in the real world. It consists in estimating in real time the pose (position + rotation) of the camera in regard to a planar marker placed into the physical environment. It was originally developed by Hirokazu Kato of Nara Institute of Science and Technology in 1999 [20]. The main characteristics of this library are the possibility to customize the targets as long as they remain square in shape, a simplified approach to calibrate the camera, the availability of several platform distributions and to be an open-source system giving access to the whole image processing algorithms.

- ArUco Markers: a binary square fiducial markers that can be used for camera pose estimation. ArUco markers are small planar targets containing 2D binary code. Each marker is encoded thanks to the grid which composes it and whose cells are colored in black and white. The ArUco recognition algorithm allows to extract, identify and estimate the pose of the different ArUco markers seen by the camera [21].

- Vuforia SDK: Uses image processing and computer vision approaches to detect and track on the y planar targets and 3D objects. It enables precise alignment of virtual objects to real-world objects. It allows to track several targets simultaneously. It is multi-platform (Windows, Android, IOs) and well suited for mobile devices [22].

- ARCore: is the platform developed by Google to provide augmented reality on mobile devices without using markers. The embedded motion tracking technology uses the phone's camera to detect and track the interesting points identified in the surrounding environment. The combination of the movement of these points with data from the phone's inertial sensors allows real-time estimation of the phone's position and orientation as it moves through space. In addition, ARCore can detect at surfaces and estimate the average lighting in its close vicinity [23].

- ARKit: allows to build high-detail AR experiences for iPad and iPhone devices. As the device moves, it maps the environment using data coming from on-board sensors (camera, gyroscope accelerometers). The inertial sensor data is fused with the camera information to accurately estimate the location of the device. In addition, ARKit can detect visual characteristics of the environment like planes and tracks motion in conjunction with information from the inertial sensors [24]

Moreover, compatibility with the Unity 3D game engine is an advantage and should be examined. Indeed, Unity 3D is currently one of the most advanced games on the market and is often used to develop industrial applications using virtual and augmented reality. For example SDKs such as Vuforia, ARCore and ARKit are fully compatible with Unity 3D.

\section{Industrial Augmented Reality Applications}

\subsection{Visualization of NDT Measurements Using Augmented Reality}

Non-destructive testing (NDT) is an analytical method used in industry to evaluate the properties of a material, component or system without employing processes that could 
destroy or affect its properties. NDT is used to detect defects within a component and determine whether the component is damaged or in need of repair. Ultrasonic transducers are often used for NDT of industrial parts in their volume or surface. This consists of emitting acoustic waves and detecting their interaction with defects present in the part. The transmitted waves, like an echo, are then converted, in real time, into a digital image of the defect thus located and characterized. One of the problems of this task concerns the visualization of the measurements in an intuitive and unequivocal way. It is clear that the use of 2D images remains insufficient to illustrate and explain the results of NDT tests. Therefore, more appropriate 3D visualization tools and methods need to be developed in order to improve the understanding and interpretation of NDT data. The objective of this project is to develop an augmented reality interface allowing visualizing directly on the part the measurements resulting from the non-destructive testing. Thus, the operator will be able to visualize the areas requiring further investigation for example. This visualization mode, which is more intuitive than simple numerical data, saves time and significantly reduces the complexity of the inspection task.

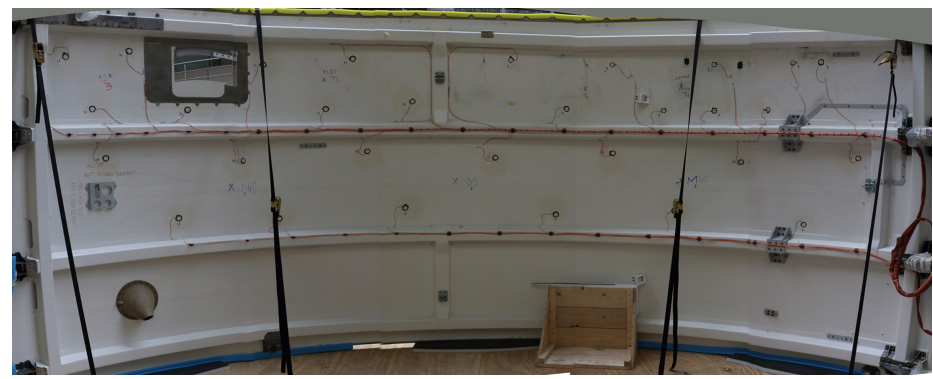

Fig. 3. Airbus A380 Nacelle

The part studied in our case is a nacelle of an Airbus A380 (Fig. 3) for which we have a set of data including:

- The nacelle CAD file (Fig. 4-a).

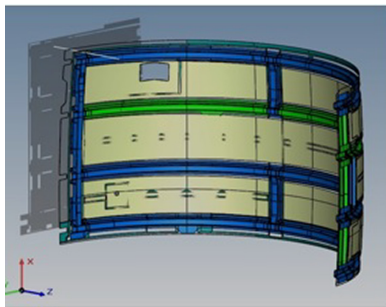

(a)

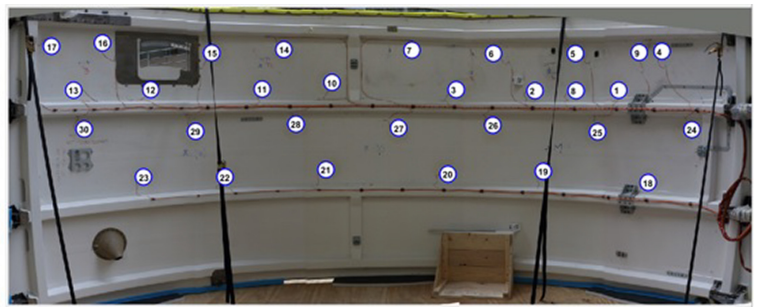

(b)

Fig. 4. Example of available data 
- The coordinates of the ultrasonic transducers and their locations on the nacelle(Fig.4-b).

- The simulation files containing defect coordinates.

Based on this data, our augmented reality application therefore aims to visualize:

- On the real structure, the sensors used for the NDT measurements.

- The structure area with a defect. Sensors whose measurements indicate the presence of a defect will delimit an area.

- The precise localization of the defect.

Our Approach. The proposed solution was developed under unity 3D and Vuforia, then deployed and tested on a tablet and a HoloLens 1 headset.

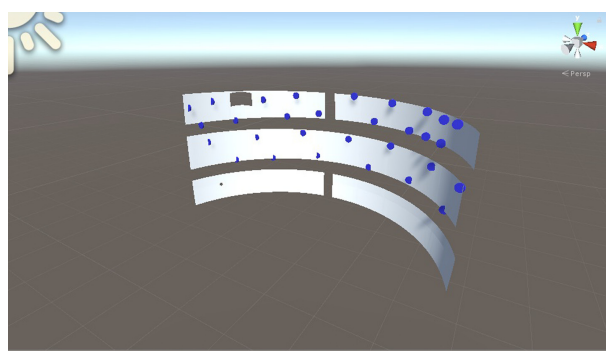

Fig. 5. 3D positioning of transducers

The first difficulty we encountered concerns the import of the nacelle 3D model under unity $3 \mathrm{~d}$. Indeed, the provided file is in Catia format which is incompatible with Unity 3D. A step of tessellation and simplification of the model was necessary to avoid overloading the computations of the augmented scene. The second step consists in creating, under 3D unity, the augmented scene. We started by positioning the sensors on the 3D model of the nacelle. It is necessary that this positioning be precise enough to allow the shown result to be coherent with the real measurements. However, the coordinates of these sensors are given in a 2D coordinate system. So, in order to represent them in 3D space, we have implemented a function that projects these positions on the surface of the nacelle whose geometry is known precisely (Fig. 5). The next step is to visualize the defect areas on the 3D model. For this purpose, we have used the NDT measurement results provided by the maintenance department, which indicate the active sensors that have detected a defect. Figure 6 shows an example of this functionality in case four sensors (symbolized here by blue dots) are active. The defect area is displayed as a polygon that passes through all four sensors. In order to reduce the defect search area, we propose to only display the areas generated by at least three and at most five active sensors.

We used Vuforia markers to implement the augmented reality part, a simple and fast solution given the complex geometry of the nacelle (large size and non-textured surface). We used Vuforia's extended tracking which, thanks to the sensors of the mobile device, continues to accurately place the virtual model in the real environment even if 


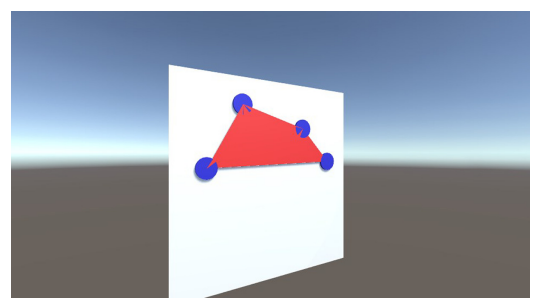

Fig. 6. Example of a defect area

the marker is lost while moving through the scene. The marker is placed in the center of the horizontal axis of the workpiece, but also on the lower part, which has no sensors. Finally, the interactive interface of the final application allows the user to select one or more sensors in order to view various information such as coordinates or temperature. The user can also choose the defect areas present in the scenario in order to visualize them in augmented reality on the real nacelle. Figure 7 shows an example of a rendering of the latest version of our application.

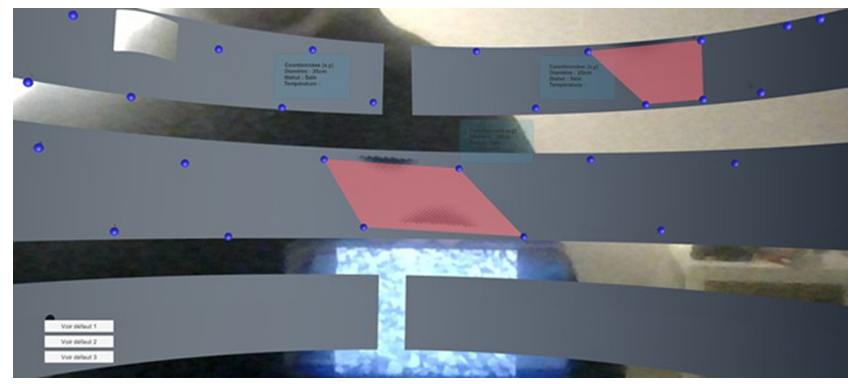

Fig. 7. View of the final version of our application

Discussion. The first experimental tests have shown that this application works well. Nevertheless, we have noticed that the tracking sometimes becomes unstable, especially when we are very close to the structure or when the marker becomes invisible. To avoid this instability, we have to be at a minimum distance of $2 \mathrm{~m}$ from the nacelle, which is not practical for the maintenance technician who has to be as close as possible to the structure. An interesting way to solve this problem is to use markerless tracking based on a simultaneous localization and mapping system (SLAM) to create a geometric reconstruction of the world while estimating the location of the camera [25, 26]. In this case, SDKs such as Google ARCore or ARKit would be more appropriate. In addition, an in-depth study of the usability of the application in real conditions will be carried out. This will enable us to obtain more refined feedback from maintenance operators, particularly on the interface's ergonomics, the affordance of augmented reality interactions, and the contribution of the proposed functionalities to the practical execution of the task. This study would make it possible to improve the application by taking into account the industrial constraints of the project. 


\subsection{Implementation of AR Application in Aeronautical Maintenance}

This project is being carried out in collaboration with Safran Landing Systems (SLS) in France. Its objective is to study the feasibility and usability of augmented reality in aeronautical maintenance. Thus, we conducted an experiment on an assembly task to observe the impact of Augmented Reality under industrial conditions. The use case corresponds to the assembly of workpieces or axles, the tightening of individual bolts and the protection of unpainted areas (Fig. 8). The participants in this experiment carried out the task under real working conditions, using two operating modes, the first one using only paper supports according to the classical process, and the second one with an augmented reality support installed on a mobile workstation. The time required to complete the task was registered and the participants' feedback was evaluated by means of questionnaires immediately after the task. We used Diota software [27] to create our augmented reality solution which was then installed on a Windows 10machine equipped with a high-definition industrial camera. This workstation is placed on a mobile trolley allowing it to be moved as required and easily installed on the assembly line. Thus, the operators involved in the experiment can test the scenario "with augmented reality" in real working conditions without being disturbed. This is important in order not to bias the results of the experiment.
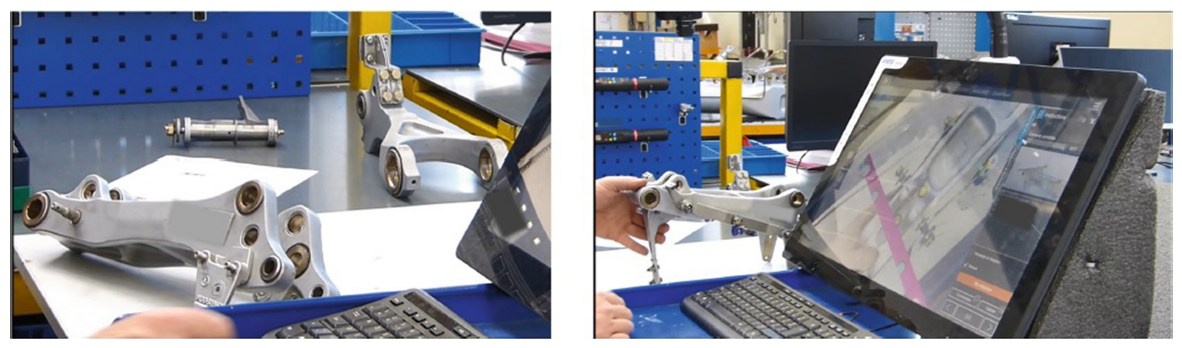

Fig. 8. The use case equipment

Measurement Protocol 9 maintenance operators were enrolled to take part in the experiment. None of them have any prior knowledge of augmented reality technologies. We took into account the level of expertise and knowledge of the participants regarding the task to be performed and aeronautical maintenance in general. We therefore divided them into three groups: Beginner, Confirmed and Expert. The participant begins the experiment using the classical procedure, which consists in searching on the paper support for the relevant information to carry out the assembly of the workpiece. The experiment is repeated with the augmented reality support. For each participant we measure the time needed to understand and achieve the task. We set up two questionnaires that the participants fill in at the end, the NASA-TLX [28] to subjectively evaluate the quality of the experience, notably the mental demand necessary to perform the task with

or without augmented reality, and the SUS [29, 30] to evaluate the degree of facility in using the augmented reality support. The analysis of the quantitative and qualitative results allows, firstly, to measure the impact of augmented reality on the performance 
of the participants, and secondly, to evaluate the usability of the augmented reality application as well as the cognitive load induced on the participants.

Discussion. The obtained results clearly demonstrate that augmented reality improves performance for all three user profiles. the "with augmented reality" scenario got the best usability score thanks to a better distribution of time between the comprehension phase and the action phase during the assembly task. We have found that augmented reality, because of its ability to facilitate the understanding of the assembly instructions, allows users to concentrate more on the action phase. This is confirmed by the decrease in the cognitive load score of the task and by a better execution time. This supports our hypothesis on the added value of augmented reality in the completion of an industrial process. Furthermore, we have noted that the impact of Augmented Reality is different depending on the level of expertise of the user. Thus, the "Beginner" users benefit the most from augmented reality which considerably reduces their cognitive load, making a strong contribution to their learning. We also noted that these augmented reality tools increase productivity when used by "Confirmed" operators but they have no effect on "Expert" users who have a perfect knowledge of the assembly process. This first experimentation has allowed us to identify and measure the added value of augmented reality on an assembly task. The obtained SUS score shows that users preferred to work with AR rather than with the reference handbook that details the maintenance process. In addition, the execution time for assembly tasks has been reduced by $74 \%$ when the AR procedure is carried out, demonstrating that AR has a direct and significant impact on the execution of maintenance tasks. The next step is to extend the experiment to other tasks related to aeronautical maintenance so that the obtained results can be generalized. You can find more details on this study in the paper we presented in 2019 at the EuroVR conference [31]. Thus, thanks to this pilot project, Safran plans to deploy Augmented Reality in other MRO departments in Asia and the Americas, and to extend it to other services such as quality control.

\section{Conclusion and Future Directions}

The purpose of this study was to present the current state of augmented reality and their practical application in the manufacturing industry. We first outlined a brief history of industrial projects that used augmented reality, we found that such technology is often used in maintenance, training and upstream design in product development. Its use continues to increase, particularly in the context of Industry 4.0, where it represents one of the cornerstones of this paradigm. Then, the paper give the most relevant hardware and software technologies of Augmented Reality actually used in industrial applications. We presented two industrial projects in which we collaborate and whose objectives are the development of augmented reality solutions for aeronautical maintenance, and the study of the usability of such technology in MRO department, taking into account industrial constraints. We have thus implemented a methodology allowing to integrate augmented reality in an industrial task and to measure its added value in a qualitative and quantitative way. Further work will consist on extending these results to evaluate augmented reality on the whole aeronautical maintenance process. Furthermore, in order to address future 
challenges for the industry 4.0 efforts should focus on improving Augmented Reality HMIs by developing new user-centric approaches that integrate workers into the design loop, and enable on-site and remote collaboration.

\section{References}

1. Kang, H.S., et al.: Smart manufacturing: Past research, present findings, and future directions. Int. J. Precis. Eng. Manuf. Green Technol. 3(1), 111-128 (2016). https://doi.org/10.1007/s40 684-016-0015-5

2. Fischer, C., Lusic, M., Faltus, F., Hornfeck, R., Franke, J.: Enabling live data controlled manual assembly processes by worker information system and near field localization system. Procedia CIRP 55, 242-247 (2016). https://doi.org/10.1016/j.procir.2016.08.013

3. Wang, X., Ong, S.K., Nee, A.Y.C.: A comprehensive survey of augmented reality assembly research. Adv. Manuf. 4(1), 1-22 (2016). https://doi.org/10.1007/s40436-015-0131-4

4. Loch, F., Quint, F., Brishtel I.: Comparing video and augmented reality assistance in manual assembly. In: 12th International Conference Intelligent Environments (IE), pp. 147-150 (September 2016)

5. Paelke, V.: Augmented reality in the smart factory: supporting workers in an industry 4.0. environment. In: IEEE Emerging Technology and Factory Automation (ETFA), pp. 1-4, (September 2016)

6. Munera, E., Poza-Lujan, J.L., Posadas-Yagüe, J.L., Simo, J., Blanes, J.F., Albertos, P.: Control kernel in smart factory environments: Smart resources integration. In: 2015 IEEE International Conference on Cyber Technology in Automation, Control, and Intelligent Systems (CYBER), pp. 2002-2005 (June 2015)

7. Caudell, T.P., Mizell, D.W.: Augmented reality: an application of heads-up display technology to manual manufacturing processes, In: 25th International Conference System Sciences, vol. 2, pp. 659-669. IEEE Press, Hawaii (1992)

8. Wohlgemuth, W., Triebfürst, G.: ARVIKA: Augmented reality for development, production and service. In: Proceedings of DARE 2000 on Designing Augmented Reality Environments, pp. 151-152, (2000)

9. Friedrich, W.: ARVIKA-augmented reality for development, production and service. In: International Symposium on Mixed Augmented Reality, pp. 3-4 (2002)

10. ARTESAS (Advanced Augmented Reality Technologies for Industrial Service Applications) Project. https://www.tib.eu/en/search/id/TIBKAT\%3A52755300X/

11. AVILUSplus. Applied Virtual Technologies Focused Long-range on the Product and Production Equipment Life Cycle. http://www.avilusplus.de

12. TESTIA, an Airbus Compagny. https://www.testia.com

13. REPLICATE - cReative-asset harvEsting PipeLine to Inspire Collective AuThoring and Experimentation. https://tev.fbk.eu/projects/replicate

14. SPARK. Spatial Augmented Reality as a Key for co-creativity. https://cordis.europa.eu/pro ject/id/688417

15. ARGO. Augmented Reality for Ground Operations. https://www.safran-group.com/media/ augmented-reality-welcome-maintenance-40-20190117

16. Zhu, J., Ong, S.K., Nee, A.Y.: A context-aware augmented reality assisted maintenance system. Int. J. Comput. Integr. Manuf. 28(2), 213-225 (2015)

17. Palmarini, R., Erkoyuncu, J.A., Roy, R., Torabmostaedi, H.: A systematic review of augmented reality applications in maintenance. Robot. Comput. Integr. Manuf. 49, 215-228 (2018)

18. Sanna, A., Manuri, F., Lamberti, F., Paravati, G., Pezzolla, P.: Using handheld devices to sup port augmented reality-based maintenance and assembly tasks. In: 2015 IEEE International Conference on Consumer Electronics (ICCE), pp. 178-179 (2015) 
19. Bach, B., Sicat, R., Beyer, J., Cordeil, M., Pfister, H.: The hologram in my hand: how effective is interactive exploration of 3D visualizations in immersive tangible augmented reality? IEEE Trans. Vis. Comput. Graph. 24(1), 457-467 (2018)

20. Kato, H., Billinghurst, M.: Marker tracking and HMD calibration for a videobased augmented reality conferencing system. In: the 2nd International Workshop on Augmented Reality, IWAR 1999, San Francisco, USA (October 1999)

21. Garrido-Jurado, S., Muñoz-Salinas, R., Madrid-Cuevas, F.J., Marin-Jiménez, M.J.: Automatic generation and detection of highly reliable fiducial markers under occlusion. Patt. Recogn. 47(6), 2280-2292 (2014)

22. Vuforia. https://developer.vuforia.com/

23. ARCore. https://developers.google.com/ar

24. ARKit. https://developer.apple.com/augmented-reality/

25. Ababsa, F.: Robust Extended Kalman Filtering for camera pose tracking using 2D to 3D lines correspondences. In: IEEE/ASME International Conference on Advanced Intelligent Mechatronics, pp. 1834-1838 (2009)

26. Maidi, M., Ababsa, F., Mallem, M.: Vision-inertial tracking system for robust fiducials registration in augmented reality. In: IEEE Symposium on Computational Intelligence for Multimedia Signal and Vision Processing, pp. 83-90 (2009)

27. Diota. Solutions 4.0 for Industry. http://diota.com/index.php/en/

28. Hart, S.G., Staveland, L.E.: Development of NASA-TLX (task load index): results of empirical and theoretical research. Adv. Psychol. 52, 139-183 (1988)

29. Brooke, J.: SUS - A Quick and Dirty Usability Scale (1996)

30. Bangor, A., Kortum, P., Miller, J.: Determining what individual SUS scores mean: adding an adjective rating scale. J. Usability Stud. 4, 114-123 (2009)

31. Loizeau, Q., Danglade, F., Ababsa, F., Merienne, F.: Evaluating added value of augmented reality to assist aeronautical maintenance workers-experimentation on on-field use case. In: Bourdot, P., Interrante, V., Nedel, L., Magnenat-Thalmann, N., Zachmann, G. (eds.) EuroVR 2019. LNCS, vol. 11883, pp. 151-169. Springer, Cham (2019). https://doi.org/10.1007/9783-030-31908-3_10 